\title{
Observation of triggering in the Milky Way
}

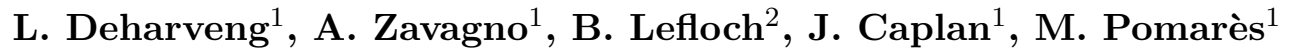 \\ ${ }^{1}$ Laboratoire d'Astrophysique de Marseille, 2 Place le Verrier, 13248 Marseille Cedex 4, France \\ email: lise.deharveng@oamp.fr \\ ${ }^{2}$ Laboratoire d'Astrophysique de l'Observatoire de Grenoble
}

\begin{abstract}
We show how the expansion of classical Galactic H II regions can trigger massivestar formation via the collect \& collapse process. We give examples of this process at work. We suggest that it also works in a turbulent medium.
\end{abstract}

Keywords. stars: formation, stars: early-type, ISM: H II regions

\section{Introduction}

How do we make a massive star? It has been shown statistically that the luminosities of young stellar objects (YSOs) correlate with the mass of their parental molecular clouds, and that the most luminous YSOs are found in molecular clouds adjacent to H II regions (Dobashi et al. 2001). Many physical processes can trigger star formation at the borders of classical H II regions (cf. the review by Elmegreen 1998). These can be divided into two main categories:

- Some of these processes assume the pre-existence of molecular condensations or inhomogeneities. For example, star formation may occur during the radiation-driven implosion of pre-existing molecular globules. This process is often advanced to explain star formation in bright rims; it has been simulated by Lefloch et al. (1994) and, more recently, by Miao et al. (2006). Also, star formation in EGGS (evaporating gaseous globules) has been proposed by Hester et al. (1998) to explain low-mass star formation in the 'pillars' of the Eagle Nebula.

- Some other processes allow formation of molecular condensations out of a nearly homogeneous medium. Among them are various types of instabilities of the ionisation front, e.g. hydrodynamical instabilities as simulated by García-Segura \& Franco (1996) and Mizuta et al. (2006). The collect \& collapse process, proposed by Elmegreen \& Lada (1977), is another such process. This is particularly interesting as it allows us to form massive condensations, and thus possibly massive objects, stars and clusters. We will now focus on this process.

We also want to stress the advantage of considering objects with a very simple morphology if we want to understand which star formation process is at work at a given location. We have selected spherical H II regions around a central exciting star, surrounded by a shell of neutral material (Deharveng et al. 2005). The MSX and the Spitzer-GLIMPSE surveys are particularly well suited for showing the presence of this material, via the emission of the associated dust. The MSX Band A at $12.3 \mu \mathrm{m}$ and the Spitzer-IRAC band at $8.0 \mu \mathrm{m}$ are dominated by the emission of polycyclic aromatic hydrocarbons (PAHs). These molecules are destroyed in the ionized gas, but ionized and excited by the far-UV photons leaking from the $\mathrm{H}$ II regions. Our selected $\mathrm{H}$ II regions are surrounded by a ring of PAH emission. An example of such a region is RCW 79 (Zavagno et al. 2006). 


\section{The collect and collapse process of star formation}

\subsection{How it works}

This process was first proposed by Elmegreen \& Lada (1977). It has been formulated analytically by Whitworth et al. (1994), and simulated by Hosokawa \& Inutsuka (2005, 2006).

Due to the high pressure of the warm ionized gas with respect to the low pressure of the cold surrounding neutral material, H II regions expand. During the supersonic expansion of an $\mathrm{H}$ II region formed around a first-generation massive star, neutral material accumulates between the ionization front and the shock front which precedes it in the neutral gas. Thus one of the signatures of the collect and collapse process is the presence of a shell of collected neutral material surrounding the H II region. This shell may be massive, up to several thousand solar masses. Later on this shell may collapse, and several massive fragments (up to a few hundred solar masses) are observed along the shell, at the periphery of the H II region. Massive objects - stars or clusters - may form inside these fragments; ultracompact (UC) second-generation H II regions may develop around these second-generation massive stars. As the stars retain the velocity of the material in which they formed, they must be observed, later on, in the direction of the parental shell; this is another signature of the collect and collapse process.

All of these signatures are observed around the Galactic H II regions Sh2-104 (Deharveng et al. 2003) and RCW 79 (Zavagno et al. 2006), demonstrating that the collect and collapse process is at work in these regions.

\subsection{Examples}

- Sh2-104 is an optical H II region excited by an O6V star. The mass of the ionized gas is $450 M_{\odot}$. The associated molecular material has been observed at IRAM, via its $\mathrm{CO}$ emission. The H II region is surrounded by a shell of molecular material formed by the matter collected during the H II region expansion. This swept-up shell has a mass $\sim 6000 M_{\odot}$. The dense material is traced thanks to the CS emission. The dense gas is mainly concentrated in four large fragments, almost regularly distributed along the shell. The brightest fragment has a mass $\sim 670 M_{\odot}$. A near-IR cluster, which is also an IRAS source of $30000 L_{\odot}$, lies in its direction. It ionizes a UC H II region.

Hosokawa \& Inutsuka (2005) have developed a model to follow the dynamical evolution of an H II region and its associated photodissociation region. Their model solves the UV and far-UV radiation transfer equations, and takes into account the thermal and chemical processes in a time dependant hydrodynamics code. They have adjusted a model to Sh2104. Assuming that Sh2-104 evolves in a homogeneous medium of $10^{3} \mathrm{~cm}^{-3}$, the model successfully reproduces the size of the H II region and the masses of the H II region and of the collected shell. According to this model, Sh2-104 is $700000 \mathrm{yr}$ old. The collected shell is predicted to be fully molecular, as observed, and gravitationally unstable.

- RCW 79 is an optical H II region, excited by a very massive star, according to its radio-continuum flux. Large amounts of dust, traced by the PAH emission at $8.0 \mu \mathrm{m}$, surround the H II region. The continuum emission of the cold dust at $1.2 \mathrm{~mm}$ has been observed with the SEST at ESO. This dust is distributed in a shell surrounding the ionized gas. This shell is fragmented. The two most massive fragments have masses in the 500-1000 $M_{\odot}$ range, and are diametrically opposite each other (a configuration observed in a number of regions). The most massive fragment harbours a near-IR cluster exciting a UC H II region. This cluster is also an IRAS point source of $55000 L_{\odot}$. Various maser sources are observed nearby, indicating that star formation is presently occurring in this region. 


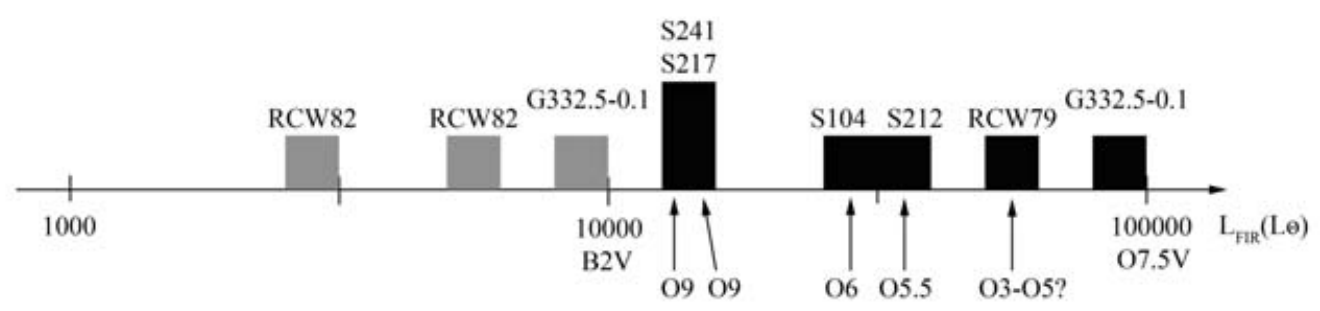

Figure 1. Far-IR luminosity of the second-generation objects formed via the collect \& collapse process at the borders of $\mathrm{H}$ II regions. $\mathrm{B} 2 \mathrm{~V}$ and $\mathrm{O} 7.5 \mathrm{~V}$ are the spectral types of stars with a luminosity of $10000 L_{\odot}$ and $100000 L_{\odot}$ respectively. The names of the $\mathrm{H}$ II regions are indicated, as well as (with arrows) the spectral types of their exciting stars, when known. Black indicates the presence of an associated UC H II region.

The shell of PAH emission surrounding the second-generation cluster is perfectly centred on the shell of PAH emission surrounding the first-generation $\mathrm{H}$ II region; this is a very strong argument in favor of the collect and collapse process.

\section{What we presently know about this process}

\subsection{The second-generation stars}

We have seen that the collect \& collapse process forms massive objects - stars and clusters. Fig. 1 gives the far-IR luminosity of the second-generation objects, in the few strong cases of formation of such objects by the collect \& collapse process. Most of these clusters contain late OB stars exciting UC H II regions.

The spectral type of the first-generation massive star is indicated in Fig. 1. Theoretically, according to Whitworth et al. (1994) no correlation is expected between the spectral type of the first- and second-generation massive stars. The dominant physical parameters which influence star formation by this process are the density of the medium into which the H II region evolves and, even more importantly, the turbulence in the swept-up shell.

Two second-generation objects observed at the borders of Sh2-212 and RCW 82 seem to be isolated massive stars. This point requires confirmation by high-sensitivity midIR observations.

\subsection{Does the collect $\&$ collapse process work in a turbulent medium?}

We have seen that shells of molecular material surround, almost entirely, the Sh2-104, and RCW $79 \mathrm{H}$ II regions. This is not the case for all of the H II regions selected as candidates for the collect \& collapse process (Deharveng et al. 2005), among which Sh2-217, Sh2-212, and Sh2-241 are different.

The CO emission maps of these regions, obtained at IRAM, show that these H II regions are presently associated with molecular filaments; they have probably formed and evolved in a turbulent medium. However, dense molecular condensations are observed at their borders (but only about half way around). Near-IR clusters, stars with near-IR excesses, UC H II regions (Sh2-217 and Sh2-212), and maser sources (Sh2-241) are observed in the direction of these molecular condensations, showing that massive-star formation is presently taking place at their borders.

Dale et al. (2005) have simulated the photoionizing feedback of a massive star born at the centre of a turbulent cloud. The simulation relating to a low-density turbulent medium shows that this radiation source is able to stop the accretion in the central 
region of the cloud, and to compress the neutral material further out in the cloud, forming condensations at the periphery of the ionized gas. The observed distribution of molecular material associated with Sh2-241 (Deharveng et al. in preparation) is strikingly similar to the distribution of the neutral material in this simulation. Thus the collect \& collapse process probably works in a turbulent medium, if this medium is not too dense and, probably, not too turbulent. These points require further study.

\section{Conclusions}

The collect \& collapse process is an efficient process for triggering massive-star formation at the borders of $\mathrm{H}$ II regions. But what is its global impact on star formation? Is this process a common way of forming stars? It may be; Hosokawa \& Inutsuka (2005) have shown theoretically that this process alone could sustain the current Galactic starformation rate. Observationally, we cannot answer at present, as this process is easy to identify only in very simple $\mathrm{H}$ II regions.

And finally, we want to stress that even the simplest $\mathrm{H}$ II regions can be very complicated! Unsharp-masked images at $8 \mu \mathrm{m}$ of the photon-dominated regions surrounding H II regions show many low-luminosity structures extending far from the ionization fronts. It shows also that the ionization fronts are very inhomogeneous. This 'leaking' structure of the ionization fronts probably also has some repercussions on star formation triggered by $\mathrm{H}$ II regions.

Additional figures are available at http://www.oamp.fr/matiere/Prague2006.html

\section{Acknowledgements}

We would like to thank all our collaborators in this long-term program, J. Brand, F. Massi, S. Kurtz, D. Nadeau, and F. Comerón. We also thank A. Whitworth and T. Hosokawa for very helpful and interesting discussions.

\section{References}

Dale, J.E., Bonnell, I.A., Clarke, C.J. \& Bate, M.R. 2005, MNRAS 358, 291

Deharveng, L., Lefloch, B., Zavagno, A., et al. 2003, A\&SA 408, L25

Deharveng, L., Zavagno, A. \& Caplan, J. 2005, A $\mathcal{E} A$ 433,565

Dobashi, K., Yonekura, Y., Matsumoto, T., et al. 2001, PASJ 53, 85

Elmegreen, B.G. \& Lada, C.J. 1977, ApJ 214, 725

Elmegreen, B.G. 1998, in: C.E. Woodward, J.M., Shull, H.A. Thronson (eds.), Origins (ASPCS), 148, p. 150

García-Segura, G. \& Franco, J. 1996, ApJ 469, 171

Hester, J.J., Scowen, P.A. \& Sankrit, R., et al. 1996, AJ 111, 2349

Hosokawa, T. \& Inutsuka, S.-I. 2005, ApJ 623, 917H

Hosokawa, T. \& Inutsuka, S.I. 2006, ApJ 646, 240

Lefloch, B. \& Lazareff, B. 1994, A\& A 289, 559

Miao, J., White, G.J., Nelson, R., et al. 2006, MNRAS 369, 143

Mizuta, A., Kane, J.O. \& Pound, M.W. 2006, ApJ 647, 1151

Whitworth A.P., Bhattal A.S., Chapman S.J., et al. 1994, MNRAS 268, 291

Zavagno, A., Deharveng, L., Comerón, F., et al. 2006, A\&A 446, 171

\section{Discussion}

BREITSCHWERDT: Why are stellar winds less important in triggering star formation than expanding HII regions? 
DeHARVENG: Stellar winds may be important in triggering star formation. I did not mention them in my presentation because they are not present or dominant in my regions (their morphologies show no signature of stellar winds).

ZINNECKER: Is it excluded that supernova remnants are present in your HII regions?

DEHARVEnG: I cannot answer for all of my regions, but most of them are classical photoionised HII regions. They are thermal radio sources, their $[\mathrm{SII}] / \mathrm{H} \alpha$ ratios are not high, we know their exciting stars... 